\title{
Diffusion of magnetic elements in a supergranular cell
}

\author{
F. Giannattasio ${ }^{1}$, M. Stangalini ${ }^{2}$, F. Berrilli ${ }^{1}$, D. Del Moro ${ }^{1}$, L. Bellot Rubio ${ }^{3}$ \\ ${ }^{1}$ Dipartimento di Fisica, Università di Roma "Tor Vergata" \\ Via della Ricerca Scientifica, 100133 Rome, Italy \\ ${ }^{2}$ INAF-Osservatorio Astronomico di Roma \\ 00040 Monte Porzio Catone (RM), Italy \\ ${ }^{3}$ Instituto de Astrofísica de Andalucía (CSIC) \\ Apdo. de Correos 3004, E-18080 Granada, Spain \\ Fabio.Giannattasio@roma2.infn.it
}

Received __ ; accepted _ 


\begin{abstract}
Small scale magnetic fields (magnetic elements) are ubiquitous in the solar photosphere. Their interaction can provide energy to the upper atmospheric layers, and contribute to heat the solar corona. In this work, the dynamic properties of magnetic elements in the quiet Sun are investigated. The high number of magnetic elements detected in a supegranular cell allowed us to compute their displacement spectrum $\left\langle(\Delta r)^{2}\right\rangle \propto \tau^{\gamma}$ (being $\gamma>0$, and $\tau$ the time since the first detection), separating the contribution of the network (NW) and the internetwork (IN) regions. In particular, we found $\gamma=1.27 \pm 0.05$ and $\gamma=1.08 \pm 0.11 \mathrm{in} \mathrm{NW}$ (at smaller and larger scales, respectively), and $\gamma=1.44 \pm 0.08$ in IN. These results are discussed in light of the literature on the topic, as well as the implications for the build up of the magnetic network.
\end{abstract}

Subject headings: Sun: photosphere 


\section{Introduction}

Magnetic field is ubiquitous in the quiet solar photosphere and interacts with plasma at all scales, from granular and mesogranular scales (see, e.g., November 1980; Roudier et al. 1998; Del Moro 2004; Berrilli et al. 2005; Centeno et al. 2007; Yelles Chaouche et al. 2011; Berrilli et al. 2013) to supergranular and giant scales (see, e.g., Hart 1956; Simon \& Leighton 1964; De Rosa \& Toomre 2004; Del Moro et al.|2007; de Wiin et al.|2008; Bellot Rubio \& Orozco Suárez 2012; Orozco Suárez \& Bellot Rubio 2012; Orozco Suárez et al. 2012; McIntosh et al. 2014). Such an interaction may give rise to magnetic reconnections and the excitation of magnetohydrodynamic (MHD) waves, which are the main mechanisms proposed to explain the heating of the solar corona (see, e.g., Alfvén 1947; Parker 1988; Hughes et al. 2003; De Pontieu et al. 2007; Tomczyk et al. 2007; van Ballegooijen et al. 2011; Stangalini et al. 2013a,b). Therefore, it is important to investigate the interaction between small-scale magnetic fields (hereafter magnetic elements) in the quiet Sun (see, e.g., Hughes et al.|2003; Viticchié et al. 2006), and between magnetic elements and plasma flows. Orozco Suárez et al. (2012) showed that magnetic elements are dispersed radially in supergranules with velocities aligned with the plasma flow.

It has been shown that the efficiency with which the magnetic elements are transported on the photosphere is well described by a power law (see, e.g., Giannattasio et al. $\mid 2013)\left\langle(\Delta r)^{2}\right\rangle=c \tau^{\gamma}$, where $\left\langle(\Delta r)^{2}\right\rangle$ is the mean square displacement, $c$ a constant, $\tau$ is the time measured since the first detection, and $\gamma$ is the spectral index. This parameter quantifies the efficiency of the transport process with respect to a random walk (RW, or normal diffusion, for which $\gamma=1$ ). When $\gamma \neq 1$ the process is termed anomalous diffusion. In particular, the regime $\gamma>1$ corresponds to a super-diffusion, while $\gamma<1$ to a sub-diffusion. Recently, several authors, both using G-band images and magnetograms from ground and space observations, agreed on a super-diffusive dynamic regime (see, e.g., Lawrence et al. 2001; Manso Sainz et al. 2011; Abramenko et al. 2011; 
Giannattasio et al. 2013; Jafarzadeh et al. 2014). In these works, magnetic elements are assumed to be passively transported by the flow.

The aim of this paper is to determine the spectral index and the diffusivity corresponding to magnetic elements within a supergranular cell, separating the contributions from network (hereafter NW) and internetwork (hereafter IN) regions, where different MHD regimes are expected. For this purpose, we take advantage of a very long (25 hours without interruption) time sequence of high resolution magnetograms, acquired in the quiet Sun by the Solar Optical Telescope (SOT) onboard Hinode.

\section{Observations and analysis}

In this work we analyzed the same Hinode/SOT data (Kosugi et al. 2007; Tsuneta et al. 2008) described in Gošić (2012) and used by Giannattasio et al. (2013) to perform their analysis on the diffusion of magnetic elements up to supergranular scales. These data belong to the Hinode Operation Plan 151 (HOP151) entitled Flux replacement in the photospheric network and internetwork, and consist of a series of magnetograms and filtergrams $25 \mathrm{hr}$ long, acquired by the Hinode Narrowband Filter Imager (NFI) in a quiet-Sun region near disk center. The series starts at 08:00:42 on 2010 November 2. The magnetogram noise level is $\sigma=6 \mathrm{G}$ for individual frames. Observations were performed at two wavelengths $\pm 160 \mathrm{~m} \AA$ from the core of the NaI D spectral line at $589.6 \mathrm{~nm}$. The pixel size is $0 " .16$ (116 km on the solar photosphere), corresponding to a spatial resolution of 0".32. The field of view (FoV) area is $\sim 51 \times 53 \mathrm{Mm}^{2}$. The cadence between successive frames (a total of 995) is $90 \mathrm{~s}$. Five-minute oscillations were filtered out by using a $k-\omega$ filter with $c_{s}=7 \mathrm{kms}^{-1}$. All the details regarding the calibration of data can be found in Gošić (2012).

In order to compute the horizontal velocity field, we used the Fast Local Correlation Tracking 


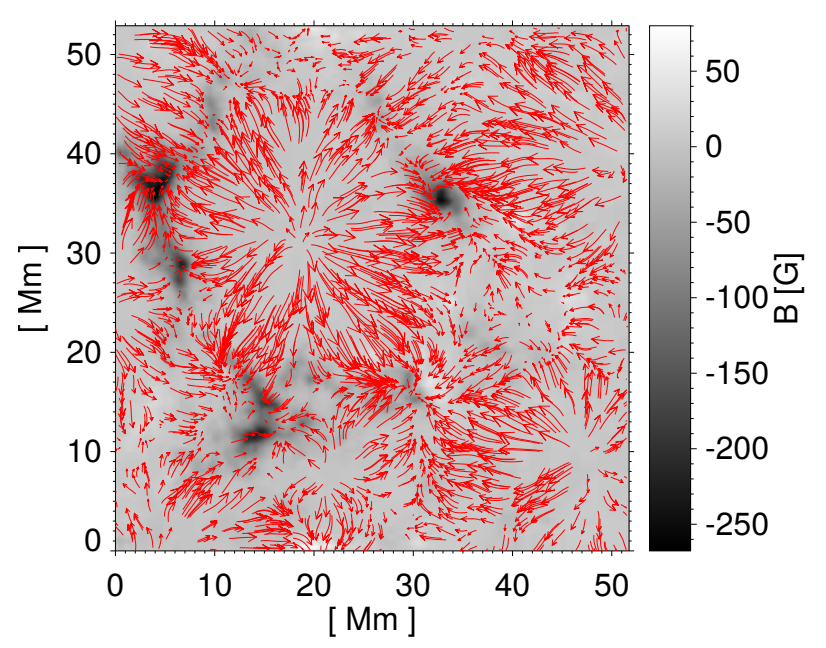

(a) Horizontal velocity field

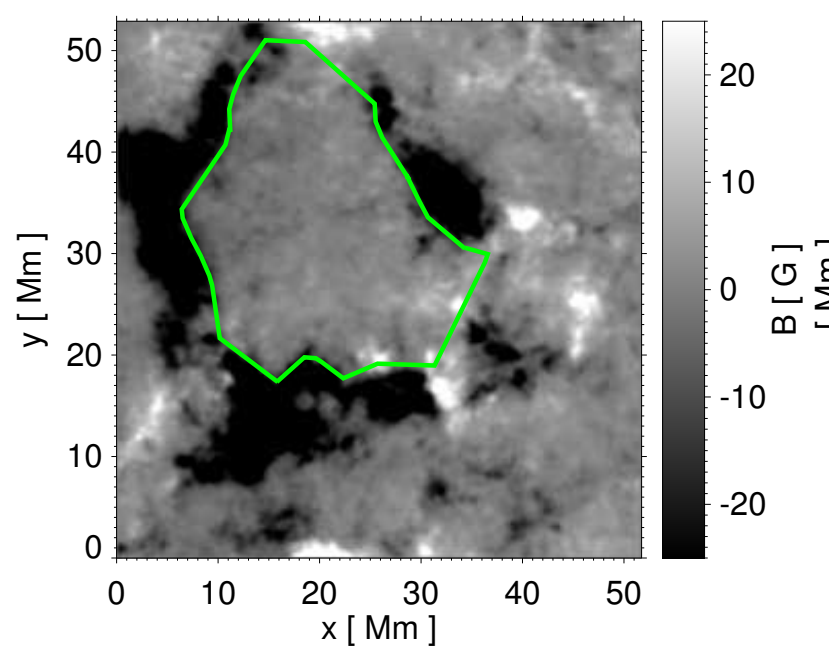

(c) Saturated mean magnetogram

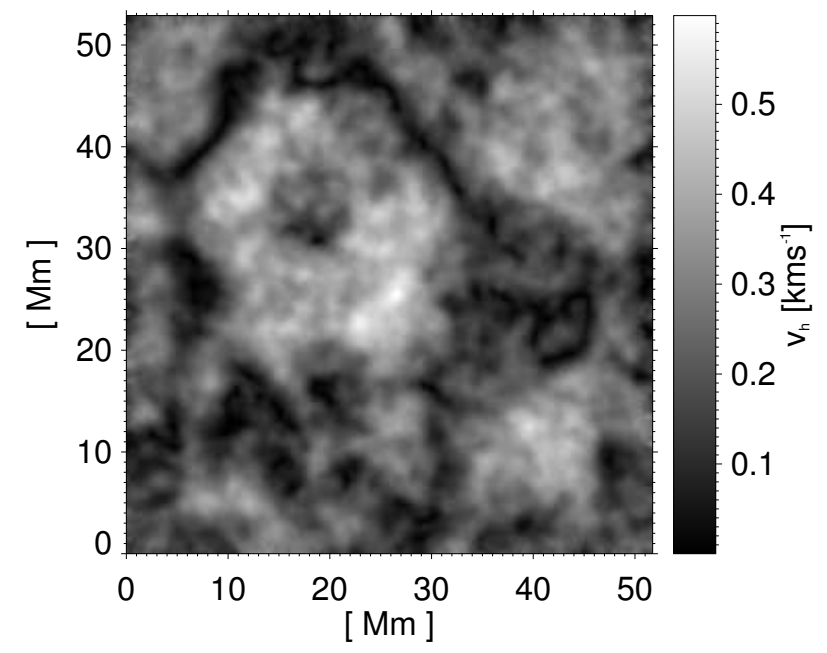

(b) Horizontal velocity amplitude

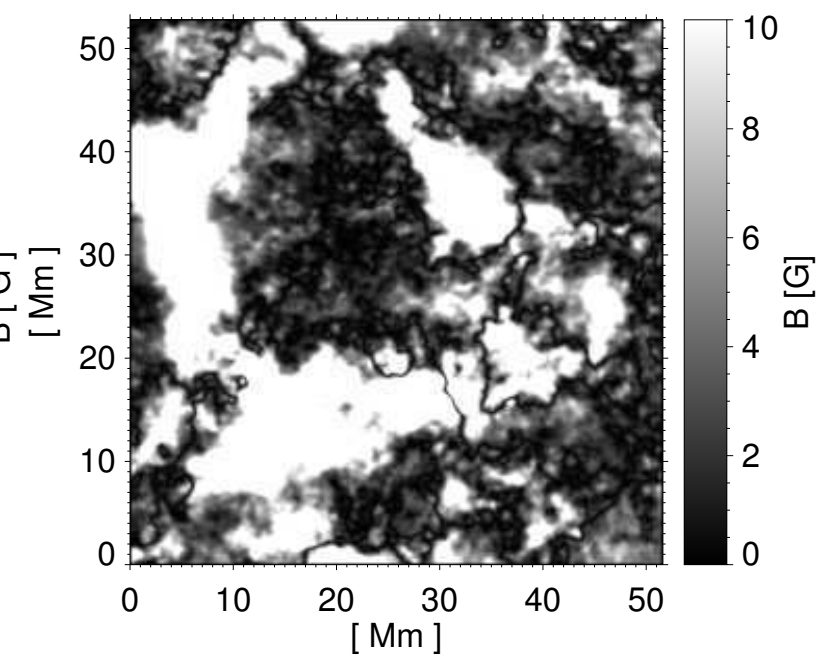

(d) Deep magnetogram

Fig. 1.- Top row: 25-hr averaged horizontal velocity field, as computed by the LCT technique, in direction (red arrows in panel a) and amplitude (panel b). The 25-hr averaged magnetogram is shown in the background on panel (a). Bottom row: average magnetogram saturated at 25 (panel c) and $10 \mathrm{G}$ (panel d). The green polygon in panel (c) highlights the supegranular cell.

code (FLCT; Fisher \& Welsch 2008), with a spatial window of 10 pixels ( $1200 \mathrm{~km})$. All the 995 velocity frames obtained were summed up to recover the 25 -hr averaged horizontal velocity field. The results of the LCT analysis are shown in Figure1 In that figure, the red arrows in panel 
(a) mark the retrieved average horizontal velocity field. In the background of the same panel we show the 25-hr averaged magnetogram. The amplitude of the horizontal velocity field is displayed in panel (b). Both the mean magnetogram and the horizontal velocity field unambiguously show a whole supergranular cell in the FoV (highlighted by the green polygon in panel (c)).

The iterative procedure described in Del Moro (2004) and used in Giannattasio et al. (2013) was then applied on the magnetograms to detect and track the magnetic elements. To avoid false detections due to time-uncorrelated signals, only those lasting at least 5 frames were considered. A total of 20145 magnetic elements was tracked in $25 \mathrm{hr}$. Their lifetime spans the range between 7.5 minutes (set by the selection criterion) and $11.1 \mathrm{hr}$.

As aforementioned, we were interested in the estimation of the diffusivity of the advected magnetic elements in a supergranular cell. For this purpose, among all the magnetic elements we considered two subsamples: i) those belonging to NW; ii) those belonging to IN. NW and IN were defined from the average magnetogram. In panel (c) of Figure 1 we show the average magnetogram saturated at $B_{s}=25 \mathrm{G}$ (4 times the noise level). The pixels adjacent to the polygon of panel (c) with $|\mathbf{B}| \geq B_{s}$ were defined to belong to NW. The pixels within the polygon and with $|\mathbf{B}|<B_{s}$ were defined to belong to IN. We found 2021 magnetic elements which are first detected, live and die within the NW; and 3563 magnetic elements which are first detected, live and die within the IN, respectively. For these subsamples we computed the mean square displacement as a function of time, $\left\langle(\Delta r)^{2}\right\rangle(\tau)$. The log-log plot of such a function, namely the displacement spectrum, consists in a line with slope $\gamma$. The diffusivities were retrieved by the relations (Monin \& Yaglom 1975; Abramenko et al. 2011)

$$
\begin{gathered}
K(\Delta r)=\frac{c \gamma}{4}\left(\frac{\Delta r^{2}}{c}\right)^{\frac{\gamma-1}{\gamma}}, \\
K(\tau)=\frac{c \gamma}{4} \tau^{\gamma-1},
\end{gathered}
$$

which describe the anomalous diffusion processes occurring in the photosphere as a function of 
both the spatial $(\Delta r)$ and temporal $(\tau)$ scales. Together with $\gamma$, also the constant $c$ was retrieved by fitting the displacement spectrum.

\section{Results}

Our data set allowed us to study the dynamic properties of the magnetic elements on a large range of spatial and temporal scales; from granular to supergranular ones. Moreover, since the FoV includes a whole supergranule, it was possible to study separately the dynamics of the NW magnetic elements and that of the IN ones in a supergranular environment.

Figure 1 shows that, on average, the horizontal velocity field inside the supergranular cell is radial, and directed from the center to the boundaries (panel (a)). The horizontal velocity is of order $\sim 10 \mathrm{~ms}^{-1}$ in the center of the cell. Moving outwards, it reaches a maximum of $\sim 600$ $\mathrm{ms}^{-1}$ at about half the cell radius, then decreases again to very low values at the boundaries (panel (b)). These results are consistent with those found by Orozco Suárez et al. (2012), who analyzed another data set belonging to HOP151.

A low magnetic flux $(\lesssim 5 \mathrm{G})$ is observed in the IN, where magnetic elements are weak and highly dynamic, standing in the same region for a shorter time than those within the NW. In order to increase the image contrast and inspect the regions with enhanced mean magnetic flux, we saturated the mean magnetogram at $10 \mathrm{G}$, obtaining the deep magnetogram in the FoV shown in panel (d) of Figure 1. The deep magnetogram also confirms the findings by Martínez González et al. (2012); Martínez Pillet (2013); Stangalini (2014), by showing a deficit of magnetic flux at the center of the supergranule at those locations where the horizontal velocity is slower. When focusing on the IN, radial structures appear which, under the hypothesis that magnetic elements are passively transported by the flow, retrace the average horizontal velocity field lines computed by LCT. In the NW, magnetic elements are stronger, more compact and larger 
in size. Due to the joint action of their augmented magnetic flux (which allows them to withstand the plasma drag force) and the slower horizontal velocity fields therein, they remain in the same regions for longer. The NW evolution takes place on several generations of elements, comparable with the supergranular time scales (about one day). On the contrary, IN magnetic elements are weaker and tend to not stay for long in the same regions.

Such a different behavior should influence the efficiency with which the magnetic field is carried along in a supergranular cell. This is important because it affects the rate of magnetic field interactions, which is a fundamental parameter to evaluate the energy with which the quiet Sun contributes to heat the upper atmosphere. To investigate this point, we computed the displacement spectrum of NW and IN magnetic elements separately.

The results are shown in Figure 2, In the IN (square symbols), the spectrum can be fitted with a single-slope power law (the blue dash-dotted line). The retrieved spectral index is $\gamma=1.44 \pm 0.08$, which corresponds to a super-diffusive regime. The uncertainties on $\gamma$ were computed as the standard deviations of the distributions obtained by randomly subsampling the magnetic elements. In the NW (diamond symbols), two power laws were used, according to the statistical method described in Main et al. (1999). We found a spectral index $\gamma=1.27 \pm 0.05$ (a super-diffusion) for temporal (spatial) scales $\tau \lesssim 600 \mathrm{~s}(\Delta r \lesssim 500 \mathrm{~km}$, the red dashed line), and $\gamma=1.08 \pm 0.11$ (which is consistent with a RW) for $\tau \gtrsim 600 \mathrm{~s}(\Delta r \gtrsim 500 \mathrm{~km}$, the red solid line). A double slope regime was first observed by Giannattasio et al. (2013) performing the same analysis on the same data set, but considering the whole FoV. The comparison with that work indicates that at small scales the IN magnetic elements give the greatest contribution to the magnetic field dynamics in the quiet Sun, while NW elements dominate at higher scales and are responsible for the change of slope.

A possible scenario to interpret these results for a superganular cell is the following. In the IN, weak magnetic elements are transported efficiently at all scales. In the NW, magnetic flux 


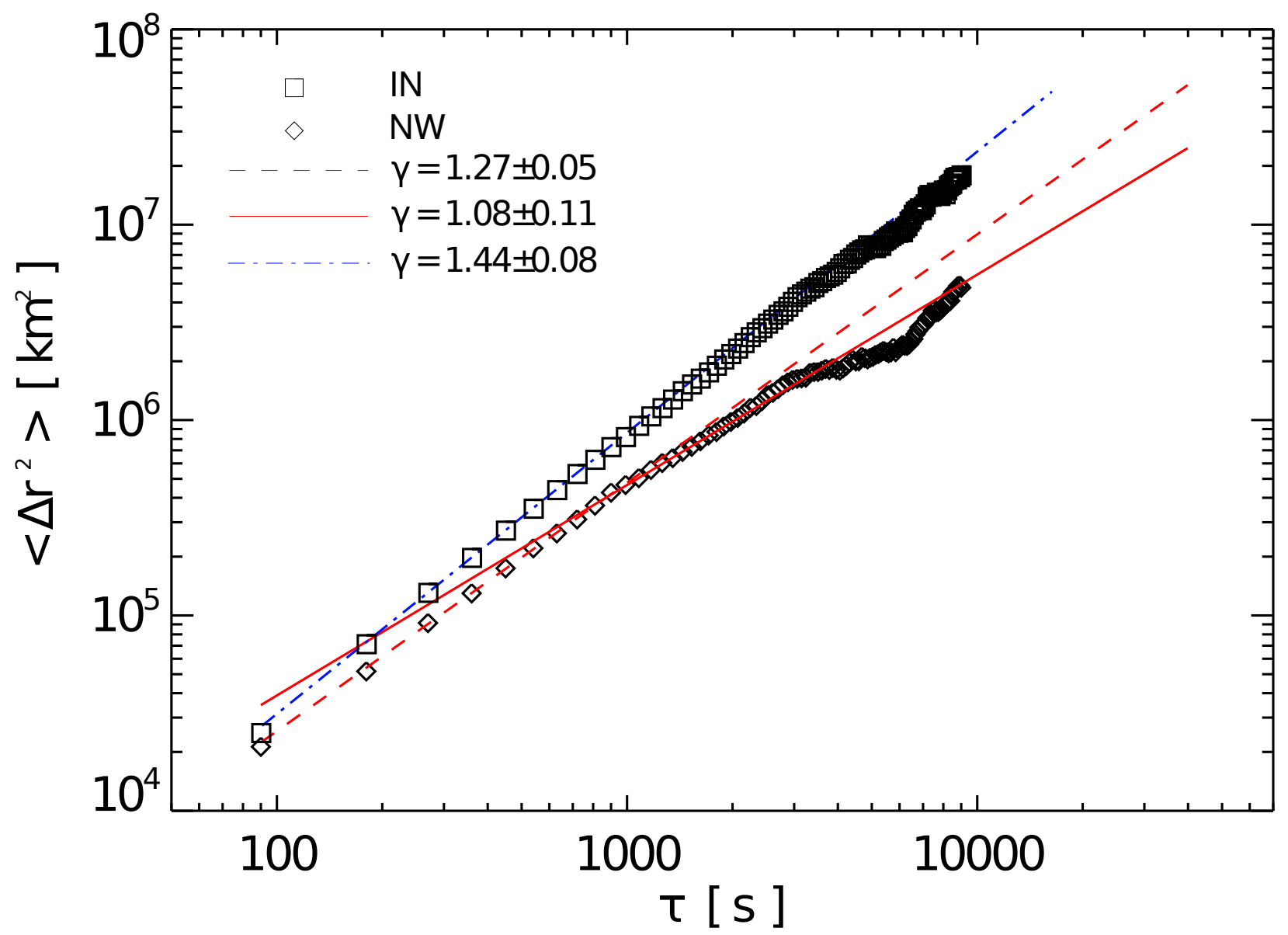

Fig. 2.- Displacement spectrum for IN (black squares) and NW (black diamonds) magnetic elements. The blue dash-dotted line fits the IN data points. The red lines fit the NW data points for $\tau \lesssim 600$ s (dashed line) and $\tau \gtrsim 600$ (solid line).

enhancement lowers the transport rate on small scales, and magnetic fields fall into velocity sinks (or traps) at a certain decorrelation mean time. From that time on, magnetic elements evolve according to the underlying velocity pattern, along more random paths. As we do not observe a change of slope in the IN regions, the velocity sinks should be more effective in the NW. Another possible interpretation implies the distinction between the strong and weak magnetic regimes observed in a supergranular cell (see Cattaneo et al. 2003). While in IN, where a weak-field regime holds, convection effectively transports the magnetic elements, in NW, where 


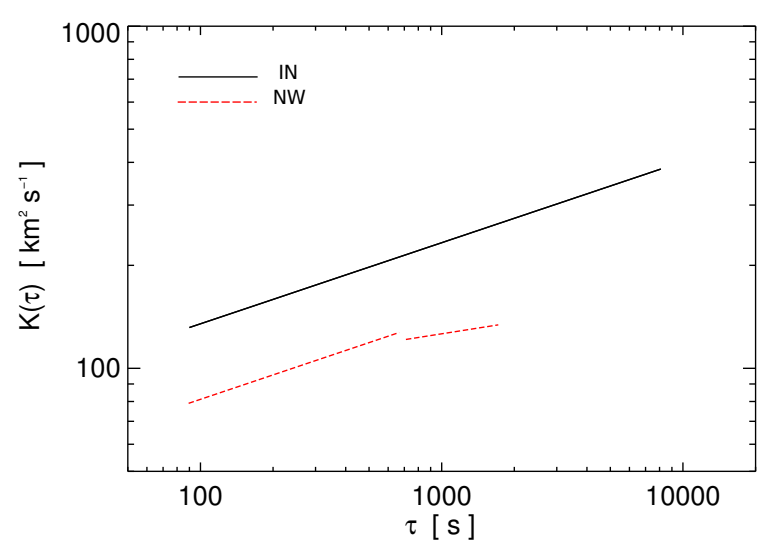

(a)

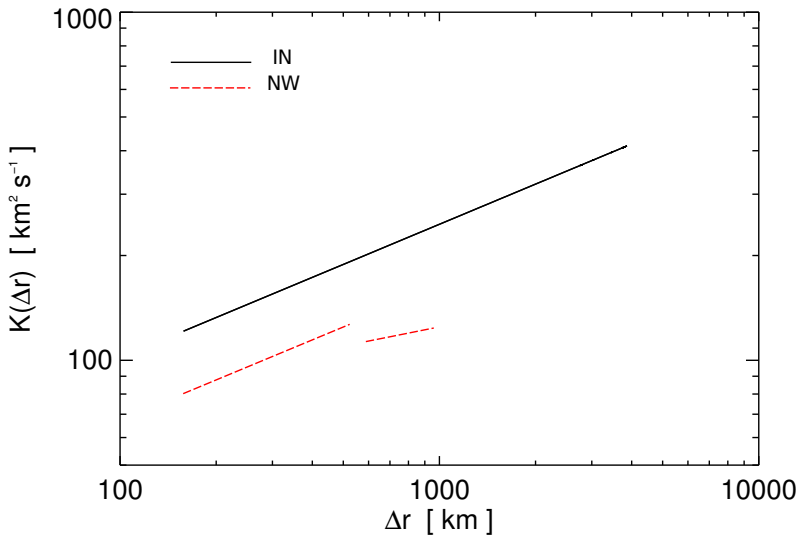

(b)

Fig. 3.- Diffusivity as a function of the temporal (panel a) and the spatial scale (panel b) for IN (black solid line) and NW (red dashed line) magnetic elements.

a strong-field regime holds, convection is reduced, to a point that magnetic elements cannot be further trasported and aggregated.

In Figure 3 we show the diffusivity $K$ as a function of the temporal (panel a) and spatial scales (panel b). In the IN the diffusivity increases from $\sim 100$ to $\sim 400 \mathrm{~km}^{2} \mathrm{~s}^{-1}$. In the NW the diffusivity increases from $\sim 80$ to $\sim 150 \mathrm{~km}^{2} \mathrm{~s}^{-1}$ for $\tau \lesssim 600 \mathrm{~s}(\Delta r \lesssim 500 \mathrm{~km})$; and much slower for longer temporal (spatial) scales. The value of the diffusivity is related to the possibility to aggregate and amplify the magnetic field at a certain scale (Abramenko et al. 2011). In the IN, the diffusivity is lower at small scales, and increases linearly with space and time due to super-diffusion. Then it follows that the amplification of the magnetic field is much more likely to happen on small scales, as it can resist to the spreading action of the flow. In the NW magnetic fields amplify more easily, as the diffusivity is a bit lower. On larger scales the difference in diffusivity between IN and NW increases more and more, especially on scales $\tau \gtrsim 600 \mathrm{~s}$ and $\Delta r \gtrsim 500 \mathrm{~km}$. Thus, in a supegranular cell the amplification at large scales should be facilitated in the NW.

The diffusion regimes found in NW and IN can help to address the following question. Does 
the magnetic field in the quiet Sun emerge in the central regions of supergranules and evolve toward the boundaries?

An analytical expression for the typical radial velocity profile expected in supergranules was found by Simon \& Weiss (1989), and successfully applied by Orozco Suárez et al. (2012) to HOP151 data. It reads $v(r)=V(r / R) \exp \left(-r^{2} / R^{2}\right), r$ being the distance from the center of the supegranule, $R$ its radius, and $V$ a free velocity parameter. By considering an upper limit for the typical velocity of $V=1 \mathrm{kms}^{-1}$ (see Orozco Suárez et al. 2012), and $R \sim 10 \mathrm{Mm}$ (found by visulal inspection of Figure 1), we can retrieve an average horizontal velocity inside the supergranule of amplitude $\bar{v}=\frac{1}{R} \int_{0}^{R} v(r) d r \simeq 0.32 \mathrm{kms}^{-1}$. This implies a crossing time (i.e., the time needed to go from the center of the supergranule to the boundaries) of $t_{\text {cross }} \gtrsim 8.7 \mathrm{hr}$. We remark that this is a lower limit value, as we have implicitly assumed a "ballistic" motion with $\gamma=2$, so that $v \sim\langle\Delta r\rangle / \tau$. According to the lifetime distribution of Giannattasio et al. (2013), only a negligible fraction of magnetic elements $(\sim 0.01 \%)$, would have a lifetime long enough to survive and reach the boundaries of the supergranular cell by starting from the center. In contrast to previous literature (see, e.g., Cattaneo et al.2003), this argument rules out the possibility for the magnetic elements which arise in the center of supergranular cells to contribute to the building up of the magnetic network. Our results are consistent with those shown in the recent work of Stangalini (2014), who found that the small scale flux emergence is not homogeneous over the supergranules, and tends to be less efficient at the centers of supergranules, and more efficient near the boundaries.

\section{Conclusions}

The rate of transport of magnetic elements in the quiet Sun is a fundamental parameter to constraint the energy supplied by the interactions between magnetic fields. Such an energy contributes to heat the solar corona. We studied the diffusion properties of magnetic elements in a supegranular cell. By LCT analysis, we found that the amplitude of horizontal velocity field is 
small in the center of the supergranular cell $\left(\sim 10 \mathrm{~ms}^{-1}\right)$, and increases toward the boundaries. A maximum is observed at about half the radius $\left(\sim 600 \mathrm{~ms}^{-1}\right)$, consistently with the most recent literature. Under the hypothesis of magnetic elements passively transported by the flow, we found a super-diffusive dynamic regime with $\gamma=1.44 \pm 0.08$ in IN; while $\gamma=1.27 \pm 0.05$ and $\gamma=1.08 \pm 0.11$ in NW, at smaller and larger scales, respectively. This confirms that different dynamic regimes are expected in supergranules. In particular, the lower diffusivity of magnetic elements in NW regions allows to amplify more easily the magnetic fields therein. We remark that for the first time we showed a variation of $\gamma$ with the horizontal velocity field in a supergranule. Finally, by a simple calculation we found that the time needed for magnetic elements to cross the supergranule from its center to the magnetic network is $\sim 8.7 \mathrm{hr}$. Only a couple of magnetic elements have a lifetime comparable with the crossing time. This suggests that magnetic elements emerging in the center of a supergranular cell cannot survive long enough to migrate to the boundaries and form the network.

This work is supported by a grant at University of Rome Tor Vergata, and by the PRIN-INAF 2010 grant, funded by the Italian National Institute for Astrophysics (INAF).

This work has benefited from discussions in the Flux Emergence meetings held at ISSI, Bern in December 2011 and June 2012.

The data used here were acquired in the framework of Hinode Operation Plan 151, entitled Flux replacement in the solar network and internetwork.

Hinode is a Japanese mission developed and launched by ISAS/JAXA, collaborating with NAOJ as a domestic partner, NASA and STFC (UK) as international partners. Scientific operation of the Hinode mission is conducted by the Hinode science team organized at ISAS/JAXA. This team mainly consists of scientists from institutes in the partner countries. Support for the post-launch operation is provided by JAXA and NAOJ (Japan), STFC (U.K.), NASA, ESA, and NSC (Norway). 


\section{REFERENCES}

Abramenko, V. I., Carbone, V., Yurchyshyn, V., et al. 2011, ApJ, 743, 133

Alfvén, H. 1947, MNRAS, 107, 211

Bellot Rubio, L. R., \& Orozco Suárez, D. 2012, ApJ, 757, 19

Berrilli, F., Del Moro, D., Russo, S., Consolini, G., \& Straus, T. 2005, ApJ, 632, 677

Berrilli, F., Scardigli, S., \& Giordano, S. 2013, Sol. Phys., 282, 379

Cattaneo, F., Emonet, T., \& Weiss, N. 2003, ApJ, 588, 1183

Centeno, R., Socas-Navarro, H., Lites, B., et al. 2007, ApJ, 666, L137

De Pontieu, B., McIntosh, S. W., Carlsson, M., et al. 2007, Science, 318, 1574

Del Moro, D. 2004, A\&A, 428, 1007

Del Moro, D., Giordano, S., \& Berrilli, F. 2007, A\&A, 472, 599

De Rosa, M. L. \& Toomre, J. 2004, ApJ, 616, 1242

de Wijn, A. G., Lites, B. W., Berger, T. E., et al. 2008, ApJ, 684, 1469

Fisher, G. H., \& Welsch, B. T. 2008, Subsurface and Atmospheric Influences on Solar Activity, 383,373

Giannattasio, F., Del Moro, D., Berrilli, F., et al. 2013, ApJ, 770, L36

Gošić, M. 2012, Master's thesis, Univ. Granada

Hart, A. B. 1956, MNRAS, 116, 38

Hughes, D., Paczuski, M., Dendy, R. O., Helander, P., \& McClements, K. G. 2003, Physical Review Letters, 90, 131101 
Jafarzadeh, S., Cameron, R. H., Solanki, S. K., et al. 2014, arXiv:1401.7522

Kosugi, T., Matsuzaki, K., Sakao, T., et al. 2007, Sol. Phys., 243, 3

Lawrence, J. K., Cadavid, A. C., Ruzmaikin, A., \& Berger, T. E. 2001, Physical Review Letters, 86,5894

Main, I. G., Leonard, T., Papasouliotis, O., Hatton, C. G., \& Meredith, P. G. 1999, Geophys. Res. Lett., 26, 2801

Manso Sainz, R., Martínez González, M. J., \& Asensio Ramos, A. 2011, A\&A, 531, L9

Martínez González, M. J., Manso Sainz, R., Asensio Ramos, A., \& Hijano, E. 2012, ApJ, 755, 175

Martínez Pillet, V. 2013, Space Sci. Rev., 178, 141

McIntosh, S. W., Wang, X., Leamon, R. J., \& Scherrer, P. H. 2014, ApJ, 784, L32

Monin, A. S., \& Yaglom, A. M. 1975, Cambridge, Mass., MIT Press, 1975.

November, L. J. 1980, PhD thesis, Colorado Univ., Boulder.

Orozco Suárez, D., \& Bellot Rubio, L. R. 2012, ApJ, 751, 2

Orozco Suárez, D., Katsukawa, Y., \& Bellot Rubio, L. R. 2012, ApJ, 758, L38

Parker, E. N. 1988, ApJ, 330, 474

Roudier, T., Malherbe, J. M., Vigneau, J., \& Pfeiffer, B. 1998, A\&A, 330, 1136

Simon, G. W. \& Leighton, R. B. 1964, ApJ, 140, 1120

Simon, G. W., \& Weiss, N. O. 1989, ApJ, 345, 1060

Stangalini, M., Solanki, S. K., Cameron, R., \& Martínez Pillet, V. 2013, A\&A, 554, A115 
Stangalini, M., Berrilli, F., \& Consolini, G. 2013, A\&A, 559, A88

Stangalini, M. 2014, A\&A, 561, L6

Tomczyk, S., McIntosh, S. W., Keil, S. L., et al. 2007, Science, 317, 1192

Tsuneta, S., Ichimoto, K., Katsukawa, Y., et al. 2008, Sol. Phys., 249, 167

van Ballegooijen, A. A., Asgari-Targhi, M., Cranmer, S. R., \& DeLuca, E. E. 2011, ApJ, 736, 3

Viticchié, B., Del Moro, D., \& Berrilli, F. 2006, ApJ, 652, 1734

Yelles Chaouche, L., Moreno-Insertis, F., Martínez Pillet, V., et al. 2011, ApJ, 727, L30 